\title{
Pulmonary and Extra Pulmonary Tuberculosis in Qatar: A First Retrospective Population-Based Study
}

\author{
Mohammed Abu Khattab', Fahmi Yousef Khan'2, Mona Al Maslamani', Abdul Latif Al-Khal1, \\ Akram El Gendy², Hussam Al Soub' ${ }^{1}$, Faraj S. Howady ${ }^{1}$ \\ ${ }^{1}$ Division of Infectious Disease, Department of Medicine, Hamad General Hospital, Doha, Qatar \\ ${ }^{2}$ Department of Medicine, Hamad General Hospital, Doha, Qatar \\ Email: mabukhattab@gmail.com
}

Received 25 September 2015; accepted 20 November 2015; published 23 November 2015

Copyright @ 2015 by authors and Scientific Research Publishing Inc.

This work is licensed under the Creative Commons Attribution International License (CC BY). http://creativecommons.org/licenses/by/4.0/

\section{Open Access}

\section{Abstract}

Background: There is limited information about extra pulmonary tuberculosis (EPTB) in Qatar. The aim of this study is to describe the demographic characteristics of patients with Tuberculosis (TB) in Qatar, and to compare the characteristics of EPTB with those of Pulmonary Tuberculosis (PTB). Patients and Methods: The study was conducted at Hamad Medical Corporation (HMC) in Qatar, from January 2005 to December 2008. It involves all cases of TB diagnosed in Qatar which do not include patients who are diagnosed by routine screening tests upon entry to state of Qatar. Results: 1221 cases of TB were reported; 654 (53.6\%) were EPTB; and 567 (46.4\%) were PTB. Nine hundred fifty-one $(\mathbf{7 7 . 9 \%})$ were males and two hundred and seventy $(22.1 \%)$ were females; the mean age was $32.7 \pm 11.7$ years; only $6.9 \%(84 / 1221)$ of them were Qataris. The most frequent associated condition was diabetesmellitus (DM) $(30 ; 2.5 \%)$, and the most frequent site involved was lymph node ( 285 cases; $43.5 \%$ ). One thousand two hundred patients continued on first-line drugs while 21 patients received second line treatment. The mean duration of antituberculous therapy was $6.7 \pm 1.7$ months. Hepatitis was the main drug toxicity. Eight hundred and forty seven patients $(69.3 \%)$ cured and eight patients $(0.7 \%)$ died, whereas the remaining three hundred and sixty-six patients $(30.0 \%)$ had left the country before completing therapy. The proportion of EPTB among females was higher than males, whereas diabetes mellitus was strongly associated with PTB. Conclusion: TB is still common in Qatar with predominance of EPTB. Although male predominated, the proportion of TB, EPTB among females was higher than males.

\section{Keywords}

Pulmonary Tuberculosis, Extra Pulmonary Tuberculosis, Tuberculosis in Qatar 


\section{Introduction}

TB remains a major global problem. In 2013, an estimated 9.0 million people developed TB, and 1.5 million died from the disease, 360,000 of whom were HIV-positive [1]. TB is slowly declining each year by an average of $1.5 \%$ a year. Moreover, the mortality rate from TB is falling and has dropped by $45 \%$ since 1990 . An estimated 37 million lives have been saved between 2000 and 2013 through effective diagnosis and treatment [1].

Qatar is a peninsula in Arabian Gulf with estimated population of 2 million in 2012, the number of economically active. Population doubled almost three times during seven years (from 44,133 in 2004 to 1,277,445 in 2011) because of that we choose this period of time to study.

This unprecedented growth resulted primarily from the recruitment of large numbers of foreign workers needed for the country's ambitious development plans. Most of this workforce is from countries with high incidence of TB which was reflected on increase total number of TB in Qatar which was increased from 184 cases in 1990 to 728 cases in 2012 with decrease in the incidence rate from 44/100,000 to of 41/100,000 respectively.

In Qatar, all cases of TB must be notified to the Communicable Disease Control and Prevention Section in the Supreme Council of Health. In 2013, the incidence rate of TB in Qatar was 40/100,000 with 511 new cases [2]. An in-depth analysis of EPTB in Qatar has not been undertaken.

Our analysis offers the first nationwide review of TB in its two forms: the pulmonary and extra pulmonary forms. It aims to provide demographic and some clinical characteristics of patients with TB in Qatar, and to compare the characteristics of EPTB with those of PTB.

\section{Method}

\subsection{Study Setting}

This retrospective population-based study was conducted at Hamad Medical Corporation (HMC) in Qatar. It is a 1500 bed public hospital. All patients with TB in Qatar are treated at HMC and the Communicable Diseases Unit at HMC keeps a registry of all cases of TB diagnosed in Qatar.

\subsection{Definitions}

The following clinical case definitions as recommended by the World Health Organization were used [1].

PTB: Two or three initial sputum smear examinations positive for AFB, or one sputum smear positive for AFB plus radiographic abnormalities consistent with active PTB, as determined by a clinician, or one sputum smear positive for AFB (smear-positive cases). Cases with three sputum smears negative for AFB but clinical and radiological features compatible with active TB and showing improvement after empirical anti-tuberculosis treatment were considered to be smear-negative cases. Both smear-positive and smear-negative patients were treated as PTB cases for our data analysis.

EPTB: This included TB of organs other than the lungs, such as lymph nodes, abdomen, genitourinary tract, skin, joints and bones, meninges, etc. Diagnosis of EPTB was based on fine needle aspiration cytology or biochemical analyses of cerebrospinal/pleural/ascetic fluid or histopathological examination or strong clinical evidence consistent with active EPTB, followed by a decision of a clinician to treat with a full course of anti-tuberculosis chemotherapy. Patients with extension of disease to organs or tissues outside the thorax, including those patients who also had pulmonary involvement, were considered as EPTB in our analysis.

\subsection{Data Collection and Analysis}

All cases of TB diagnosed from January 2005 to December 2008 were identified using the Communicable Diseases Unit registry. For each patient, demographic data including age, sex, nationality, and clinical characteristics were recorded. Clinical characteristics included, co-morbid conditions (diabetes mellitus, HIV etc.), treatment history (use of immunosuppressive drugs, steroids), past history of treatment for TB, history of contact with a case of TB, laboratory and radiographic results were obtained using the medical records and the electronic system.

Statistical analysis was performed with SPSS software (v 21; IBM Corp, Armonk, NY, USA). Quantitative variables were expressed as mean, standard deviation (SD) and range. The student $t$-test was used for continuous variables, and the Mann-Whitney $U$ test was used if the quantitative variables were not normally distributed. 
Fisher exact test or Chi-Square test was used to compare PTB and EPTB patients in relation to Age, sex difference, co-morbidities, and history of contact with the sick patients. Results were considered significant if the P-value is less than 0.05 .

\section{Results}

Between 2005 and 2008, 1221 cases of tuberculosis were reported to TB clinic at Al-Doha city, 654 (53.6\%) were EPTB and 567 (46.4\%) were PTB. Nine hundred fifty one (77.9\%) were males and two hundred seventy (22.1\%) were females (Male:Female ratio was 3.51:1); the mean age was $32.7 \pm 11.7$ years (2 - 84 years), and only 6.9\% (84/1221) of them were Qataris [Table 1]. Among patients with EPTB, the mean age was $31.95 \pm$ 11.06 and the male to female ratio was 3.36:1.

Of the 1221 patients, 147 (14.3\%) had DM, 30 (2.5\%) had prior history of TB, 12 (1.0\%) had history of contact with TB cases and two $(0.2 \%)$ patients were HIV infected.

The most frequently reported forms of EPTB were tuberculous lymphadenitis (285 cases, 43.5\%) and pleural TB (207 cases, 31.6\%) [Table 2].

Initially all 1221 patients received a combination of Isoniazid, Rifampicin, Pyrazinamide, and either Ethambutol or Streptomycin. One thousand two hundred patients continued on first line drugs while 21 patients

Table 1. Demographic data of the 1221 patients.

\begin{tabular}{cc} 
Variable & Number of patients (\%) \\
Gender & \\
Male & $951(77.9)$ \\
Female & $270(22.1)$ \\
Male:female ratio & $3.15: 1$ \\
Age (mean \pm SD) & $32.7 \pm 11.7(2-84$ year) \\
Age group & \\
$0-14$ & $22(1.8 \%)$ \\
$15-24$ & $293(24.0 \%)$ \\
$25-34$ & $474(38.8 \%)$ \\
$35-44$ & $193(15.8 \%)$ \\
$\geq 45$ & $6(19.6 \%)$ \\
Nationality & $84(6.9 \%)$ \\
Qatari & $1173(93.1 \%)$ \\
Non-Qatari & \\
\hline
\end{tabular}

Table 2. Frequency of reported tuberculosis cases by site of disease.

\begin{tabular}{cc|}
\hline Variable & Number of patients (\%) \\
\hline Pulmonary TB & $567(46.4 \%)$ \\
Extra pulmonary TB & $654(53.6 \%)$ \\
Lymph node & $285(43.5 \%)$ \\
Pleura & $207(31.6 \%)$ \\
Abdomen & $49(7.5 \%)$ \\
Meningeal & $30(4.6 \%)$ \\
Miliary & $17(2.6 \%)$ \\
Genitourinary & $9(1.4 \%)$ \\
Osteoarticular & $2(0.3 \%)$ \\
Others & $58(13.0 \%)$
\end{tabular}


received second line treatment mainly due to first line side effects since we have low incidence of MDR Tb in Qatar 1.2\% (0.34 - 3.1) [2]. First-line drug susceptibility testing is done for all culture positive patients. Secondline drug susceptibility test for MDR is done at the Mayo Clinic Reference Laboratory.

The mean duration of antituberculous therapy was $6.7 \pm 1.7$ months (3 - 24 months).

Drug toxicity was reported as following: hepatitis in 131 (10.7\%) cases, Hyperuricemia 83 (6.8\%) cases, skin rash in 33 (2.7\%) patients, blurring of vision in $9(0.7 \%)$ and peripheral neuritis in $5(0.4 \%)$.

Eight hundred forty seven patients $(69.3 \%)$ cured and eight patients $(0.7 \%)$ died, whereas the remaining three hundred sixty six patients (30.0\%) had left the country before completing therapy.

A comparative analysis between PTB and EPTB is described in [Table 3].

\section{Discussion}

The proportion of EPTB among all the reported TB cases exhibits wide variation and has increased in different populations. In Qatar, EPTB is increasing, accounting for 53.6\% of all reported TB cases in the current study compared with only $29 \%$ in 2005 [3]. This high percentage is not comparable with other reports [4]-[8]. These differences may be attributable to the difference in socio-demographic characteristic of our patients or due to success in early detection of more cases of EPTB due to presence of advanced diagnostic facilities and effective reporting system in Qatar.

In agreement with many reports from Nepal, Turkey, USA, South Africa, China and Saudi Arabia [4]-[8], our study showed a significant male predilection compared to female patients. In some areas of Pakistan, Bangladesh and Afghanistan [9]-[11]. EPTB showed female preponderance.

The site of organ involvement in EPTB varies from study to study. Some studies show the lymph nodes to be the most frequent form of EPTB; whereas in other studies pleurae were found to be involved most frequently [12]-[15]. The reason for these variations is unclear. In our study, the most frequent site was the lymph nodes followed by the pleura. An analytic comparison between EPTB and PTB showed that young age is associated with EPTB, but DM is strongly associated with PTB $(\mathrm{P}<0.001)$. A significant difference was also observed between PTB and EPTB cases; the proportion of EPTB among females is higher than males $(\mathrm{P}<0.001)$. This is consistent with many reports worldwide [16]-[21].

We noticed low incidence of HIV in our study (two (0.2\%) patients) that is mainly due to our registry only include patients whom are Nationals or expatriates with a resident permit in Qatar and HIV screening has been incorporated in pre-employment, pre-marital and preresidency permit procedures for everyone along with screening for all blood donors in the blood bank, all women in the women hospital upon their first visit and with every pregnancy, all drug users referred for psychiatric treatment in the psychiatric hospital and students travelling abroad to attend university are also offered and encouraged to test. The vast majority of the population of Qatar has tested for HIV at least once in the last few years. Generally Qatar remains a low prevalence country with a steady rate of new HIV infections diagnosed every year (less than 10 new cases diagnosed per year). In 2013, only 18 new cases were reported in spite of that over 500,000 HIV test/year done in Qatar [22].

The mean duration of antituberculous therapy was $6.7 \pm 1.7$ months (3 - 24 months) short treatment duration was mainly due to some of patients (three hundred sixty six patients $(30.0 \%)$ had left the country back to their home country without competition of the medication and since we do not have a link with the health authorizes in those countries to follow up those patients we cannot determine the final treatment duration, so the treatment duration mentioned here reflects duration that was completed in Qatar before those patient left.

The most important limitation of our study was its retrospective nature; the fact that didn't allow us to study many variables such as co-morbidities, social factors in details as the medical records were incomplete in many occasions.

Table 3. Comparison of selected demographic data for EPTB and PTB.

\begin{tabular}{ccccc}
\hline & EPTB & PTB & P value & 0.01 \\
Age & $31.95 \pm 11.06$ & $33.63 \pm 12.36$ & $<0.001$ \\
Sex $(\mathrm{M} / \mathrm{F})$ & $504 / 150$ & $447 / 120$ & $<0.001$ \\
Diabetes mellitus & $46(7 \%)$ & $128(22 \%)$ & 0.51 \\
Contact history & $6(0.9 \%)$ & $6(1 \%)$ & \\
\hline
\end{tabular}




\section{Conclusions}

In conclusion, TB is still common in Qatar with predominance of EPTB. Although male predominated, the proportion of EPTB among females was higher than males. Lymphadenitis is the most common form of EPTB in our region and diabetes was the most associated condition.

Results of our study suggest that younger age may be independent risk factors for EPTB, relative to PTB in Qatar. While diabetes mellitus was found as a significant risk factor for PTB in our study.

However, based on our results TB control programs might usefully target young populations for early diagnosis of EPTB to decrease TB morbidity and mortality and it is important to periodically screen the patients with chronic conditions like diabetes for occurrence of tuberculosis with active treatment for latent infections trying to reduce incidence of TB in those population.

Further studies are needed in other high-burden countries to confirm our findings.

We suggest a better and effective communication between different health authorities specially between countries with high burden of TB and other countries in notification and follow up of active cases whom were diagnosed abroad to ensure appropriateness of treatment regimens and duration.

\section{Conflict of Interest}

No competing interest declared.

\section{Ethical Approval}

The study was approved by the research committee of the Hamad Medical Corporation.

\section{References}

[1] (2015) Global Tuberculosis Report. World Health Organization. http://www.who.int/tb/publications/global_report/gtbr2015_executive_summary.pdf?ua=1

[2] WHO (2014) Tuberculosis Country Profiles. https://extranet.who.int/sree/Reports?op=Replet\&name=\%2FWHO_HQ_Reports\%2FG2\%2FPROD\%2FEXT\%2FTBC ountryProfile\&ISO2=QA\&LAN=EN\&outtype=html

[3] Al Marri, M., Al Hail, L., Al Otaibi, S. and Al Marri, N.D. (2006) The Time of Reactivation of Tuberculosis in Expatriates in the State of Qatar. Qatar Medical Journal, 15, 21-23.

[4] Sreeramareddy, C.T., Panduru, K.V., Verma, S.C., Joshi, H.S. and Bates, M.N. (2008) Comparison of Pulmonary and Extra Pulmonary Tuberculosis in Nepal-A Hospital-Based Retrospectivestudy. BMC Infectious Diseases, 8, 8. http://dx.doi.org/10.1186/1471-2334-8-8

[5] Llgazli, A., Boyaci, H., Basyigit, L. and Yildiz, F. (2004) Extrapulmonary Tuberculosis: Clinical and Epidemiologic Spectrum of 636 Cases. Archives of Medical Research, 35, 435-441. http://dx.doi.org/10.1016/j.arcmed.2004.05.008

[6] Peto, H.M., Pratt, R.H., Harrington, T.A., LoBue, P.A. and Armstrong, L. (2009) Epidemiology of Extrapulmonary Tuberculosis in the United States, 1993-2006. Clinical Infectious Diseases, 49, 1350-1357. http://dx.doi.org/10.1086/605559

[7] Wang, X., Yang, Z., Fu, Y., Zhang, G., Wang, X., Zhang, Y. and Wang, X. (2014) Insight to the Epidemiology and Risk Factors of Extrapulmonary Tuberculosis in Tianjin, China during 2006-2011. PLoS ONE, 9, e112213. http://dx.doi.org/10.1371/journal.pone.0112213

[8] Al-Otaibi, F. and El Hazmi, M.M. (2010) Extra-Pulmonary Tuberculosis in Saudi Arabia. Indian Journal of Pathology \& Microbiology, 53, 227-231. http://dx.doi.org/10.4103/0377-4929.64327

[9] Ullah, S., Shah, S.H., Aziz-ur-Rehman, Kamal, A., Begum, N. and Khan, G. (2008) Extrapulmonary Tuberculosis in Lady Reading Hospital Peshawar, NWFP, Pakistan: Survey of Biopsyresults. Journal of Ayub Medical College Abbottabad, 20, 43-46.

[10] Karim, M.M., Chowdhury, S.A., Hussein, M.M. and Faiz, M.A. (2006) A Clinical Study on Extrapulmonary Tuberculosis. Journal of Bangladesh College of Physicians and Surgeons, 24, 19-28.

[11] Fader, T., Parks, J., Khan, N.U., Manning, R., Stokes, S. and Nasir, N.A. (2010) Extrapulmonary Tuberculosis in Kabul, Afghanistan: A Hospital-Based Retrospective Review. International Journal of Infectious Diseases, 14, e102e110. http://dx.doi.org/10.1016/j.ijid.2009.03.023

[12] Arstaedt, A.S. (2013) Extrapulmonary Tuberculosis among Adults: Experience at Chris Hani Baragwanath Academic Hospital, Johannesburg, South Africa. South African Medical Journal, 104, 22-24. http://dx.doi.org/10.7196/samj.6374 
[13] Gomes, T., Reis-Santos, B., Bertolde, A., Johnson, J.L., Riley, L.W. and Maciel, E.L. (2014) Epidemiology of Extrapulmonary Tuberculosis in Brazil: A Hierarchical Model. BMC Infectious Diseases, 14, 9. http://dx.doi.org/10.1186/1471-2334-14-9

[14] Noertjojo, K., Tam, C.M., Chan, S.L. and Chan-Yeung, M.M.W. (2002) Extra-Pulmonary and Pulmonary Tuberculosis in Hong Kong. International Journal of Tuberculosis and Lung Disease, 6, 879-886.

[15] Rowińska-Zakrzewska, E., Korzeniewska-Koseła, M. and Roszkowski-Śliż, K. (2013) Extrapulmonary Tuberculosis in Poland in the Years 1974-2010. Pneumonologia i Alergologia Polska, 81, 121-129.

[16] Gunal, S., Yang, Z., Agarwal, M., Koroglu, M., Arıcı, Z.K. and Durmaz, R. (2011) Demographic and Microbial characteristics of Extrapulmonary Tuberculosis Cases Diagnosed in Malatya, Turkey, 2001-2007. BMC Public Health, 11, 154. http://dx.doi.org/10.1186/1471-2458-11-154

[17] Wang, X., Yang, Z., Fu, Y., Zhang, G., Wang, X., Zhang, Y. and Wang, X. (2014) Insight to the Epidemiology and Risk Factors of Extrapulmonary Tuberculosis in Tianjin, China during 2006-2011. PLoS ONE, 9, e112213. http://dx.doi.org/10.1371/journal.pone.0112213

[18] Ducomble, T., Tolksdorf, K., Karagiannis, I., Hauer, B., Brodhun, B., Haas, W. and Fiebig, L. (2013) The Burden of Extrapulmonary and Meningitis Tuberculosis: An Investigation of National Surveillance Data, Germany, 2002 to 2009. Eurosurveillance, 18, pii: 20436.

[19] Prakasha, S.R., Suresh, G., D’sa, I.P., Shetty, S.S. and Kumar, S.G. (2013) Mapping the Pattern and Trends of Extrapulmonary Tuberculosis. Journal of Global Infectious Diseases, 5, 54-59. http://dx.doi.org/10.4103/0974-777X.112277

[20] Ozvaran, M.K., Baran, R., Tor, M., Dilek, I., Demiryontar, D., Arinc, S., Toker, N., Chousein, E.U. and Sogukpinar, O. (2007) Extrapulmonary Tuberculosis in Non-Human Immunodeficiency Virus-Infected Adults in an Endemic Region. Annals of Thoracic Medicine, 2, 118-121. http://dx.doi.org/10.4103/1817-1737.33700

[21] Musellim, B., Erturan, S., Sonmez Duman, E. and Ongen, G. (2005) Comparison of Extra-Pulmonary and Pulmonary Tuberculosis Cases: Factors Influencing the Site of Reactivation. International Journal of Tuberculosis and Lung Disease, 9, 1220-1223.

[22] http://www.unaids.org/sites/default/files/country/documents//QAT_narrative_report_2014.pdf 